\title{
The response of laser interferometers to a gravitational wave
}

\author{
Adrian Melissinos ${ }^{a}$ and Ashok Das ${ }^{a, b}$ \\ ${ }^{a}$ Department of Physics and Astronomy, University of Rochester, Rochester, NY 14627-0171, USA and \\ ${ }^{b}$ Saha Institute of Nuclear Physics, 1/AF Bidhannagar, Calcutta 700064, India
}

\begin{abstract}
Laser interferometer detectors are now widely used in an attempt to detect gravitational waves $(\mathrm{gw})$. The interaction of the gw with the light circulating in the interferometer is usually explained in terms of the motion of the "free" mirrors that form the interferometer arms. It is however instructive to show that the same result can be obtained by simply calculating the propagation of an electromagnetic plane wave between "free mirrors" in the curved space-time induced by the gw. One finds that the plane wave acquires frequency modulation sidebands at the gw frequency, as would be expected from the absorption and emission of gravitons from and to the gw. Such sidebands are completely equivalent to the time-dependent phase shift imposed on the plane wave, that follows from the conventional calculation.
\end{abstract}

\section{INTRODUCTION}

In discussing the operation of gravitational wave (gw) interferometric detectors [1, 2] it is usually stated that the distance between the beam splitter and the mirrors at the end of the arms is changed by the gw [3, 44]. When light beams propagate in the arms, the change in the proper length of the two arms results in a phase shift between the light beams that return to the beam splitter. The phase shift is the physical observable that indicates the presence of a gw. The above statement is valid if the mirrors are free to move (along the axis of the arms) and it is expressed in the laboratory frame of reference, what is referred to as the Local Lorentz (LL) gauge. It is however much easier to calculate the resulting phase shift in the Transverse Traceless (TT) gauge. In the TT gauge the gw has a very simple form and the coordinates of free particles (i.e. of the mirrors) are not changed in the presence of the gw, even though their separation does change [5, 6].

The geometry of space is defined by the infinitesimal interval [7]

$$
d s^{2}=g_{\mu \nu} d x^{\mu} d x^{\nu}, \quad \mu, \nu,=0,1,2,3,
$$

where summation over repeated indices is implied. For weak fields we write the metric tensor as

$$
g_{\mu \nu}=\eta_{\mu \nu}+h_{\mu \nu}, \quad h_{\mu \nu} \ll 1 .
$$

The flat-space (Minkowski) metric $\eta_{\mu \nu}$ is chosen to be

$$
\eta_{\mu \nu}=\left(\begin{array}{cccc}
-1 & 0 & 0 & 0 \\
0 & 1 & 0 & 0 \\
0 & 0 & 1 & 0 \\
0 & 0 & 0 & 1
\end{array}\right)
$$

Consider the interferometer in the $x-y$ plane and that a gravitational wave of angular frequency $\Omega$ is incident along the $z$-axis. In the TT gauge the potential $h_{\mu \nu}$ is given by the real part of the following expression

$$
h_{\mu \nu}=e^{-i\left(\Omega t+k_{\Omega} z\right)}\left(\begin{array}{cccc}
0 & 0 & 0 & 0 \\
0 & h_{+} & h_{\times} & 0 \\
0 & h_{\times} & -h_{+} & 0 \\
0 & 0 & 0 & 0
\end{array}\right) .
$$

$h_{+}$and $h_{\times}$are the (real) amplitudes of the "parallel" and "cross" polarization states of the gw. To be specific we will place the origin of the coordinates at the beam splitter and orient the interferometer arms along the $x$ and $y$ - axes, as shown in Fig. (1). In the next section we calculate, in the TT gauge, the round trip time for the propagation of light from the beam splitter to the end mirror and back to the origin, in the presence of a gw. We will then obtain the same result by considering, again in the TT gauge, the direct interaction of the gw with the light circulating in the arms.

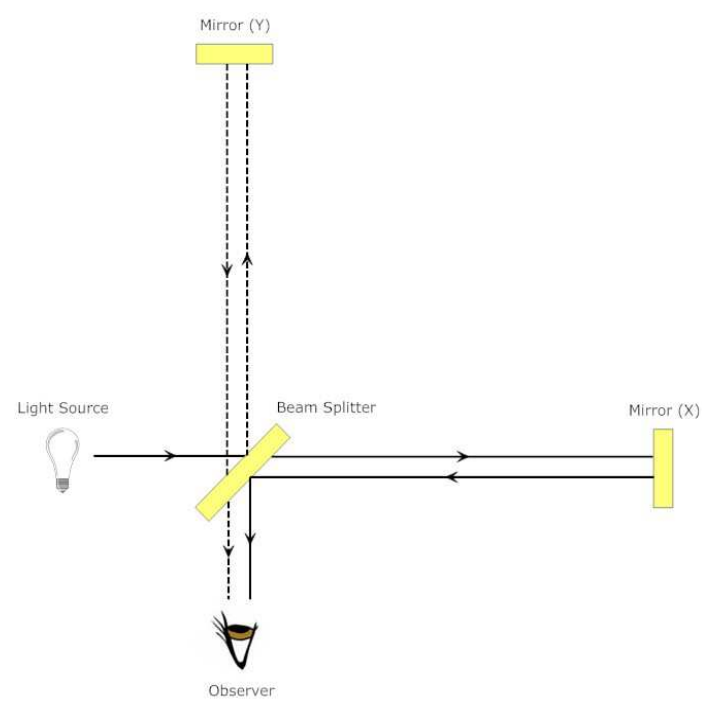

FIG. 1. Configuration of a simple Michelson Interferometer. 


\section{CONVENTIONAL CALCULATION}

We will work in the TT gauge so the coordinates of the mirrors are unchanged by the presence of the gravitational wave. We will calculate the time it takes for light leaving the beam splitter (the origin) to reach the end mirror, where it is reflected, and to return to the the beam origin. For light $d s^{2}=0$, and since the propagation is in the $x-y$ plane, Eq. (1) reduces to

$$
g_{00} c^{2} d t^{2}+g_{11} d x^{2}+g_{22} d y^{2}+2 g_{12} d x d y=0
$$

For propagation only along the $x$-axis, and since we can set $z=0$,

$$
c\left|\frac{d t}{d x}\right|=\left|\frac{g_{11}}{g_{00}}\right|^{1 / 2}=\left(1+h_{11}(t)\right)^{1 / 2} \simeq 1+\frac{1}{2} h_{+} e^{-i \Omega t}
$$

The time interval for a round trip is obtained by integrating from the origin to the end of the arm, $x=L$, and back to the origin. If the light leaves at $t=t_{0}$ and returns at $t=t_{r}$

$$
t_{r}=t_{0}+\int_{0}^{L}\left|\frac{d t}{d x}\right| d x-\int_{L}^{0}\left|\frac{d t}{d x}\right| d x,
$$

where the minus sign before the second integral accounts for the fact that on the return trip $d t / d x=-|d t / d x|$. In carrying out the integration we can replace $t$ in the exponential by $(x / c)$, because any corrections will be of second order in $h$. We also define the one way travel time in the absence of the gw as $T=L / c$. Thus

$$
\begin{aligned}
t_{r}=t_{0} & +\frac{1}{c} \int_{0}^{L}\left[1+\frac{h_{+}}{2} e^{-i \Omega\left(t_{0}+x / c\right)}\right] d x \\
& -\frac{1}{c} \int_{L}^{0}\left[1+\frac{h_{+}}{2} e^{-i \Omega\left(t_{0}+2 T-x / c\right)}\right] d x \\
=t_{0} & +\frac{2 L}{c}+\frac{h_{+}}{2 i \Omega} e^{-i \Omega t_{0}}\left[1-e^{-i 2 \Omega T}\right] \\
=t_{0} & +\frac{2 L}{c}+h_{+} T e^{-i \Omega\left(t_{0}+T\right)} \frac{\sin \Omega T}{\Omega T}
\end{aligned}
$$

This suggests that we can write

$$
h_{11}\left(t_{r}\right)=h_{+} e^{-i \Omega t_{r}} \simeq h_{+} e^{-i \Omega\left(t_{0}+2 T\right)},
$$

where we have neglected higher order terms in the metric. As a result, the travel time along the $x$-axis can be expressed as

$$
\Delta t_{x}\left(t_{r}\right)=t_{r}-t_{0}=\frac{2 L}{c}+h_{11}\left(t_{r}\right) \frac{L}{c} \frac{\sin (\Omega T)}{\Omega T} e^{i \Omega T} .
$$

For the $y$-axis we obtain the same result with $h_{11}(t)$ replaced by $h_{22}(t)=-h_{11}(t)$. Thus the difference in travel time between the two arms is

$$
\Delta t(t)=\Delta t_{x}(t)-\Delta t_{y}(t)=2\left[h_{+} e^{-i \Omega t}\right] \frac{L}{c} \frac{\sin (\Omega T)}{\Omega T} e^{i \Omega T}
$$

A difference in the time of arrival of the light from the two arms, implies a phase shift between the two fields. If the angular frequency of the carrier is $\omega_{0}$, the phase shift corresponding to a delay $\Delta t$, is $\Delta \phi=\omega_{0} \Delta t=c k_{0} \Delta t$, where $k_{0}=\omega / c$ is the wavenumber of the carrier. Therefore when the gw is normally incident on the interferometer plane and when the polarization of the $\mathrm{gw}$ is along the interferometer axes, the observable phase shift is

$$
\Delta \phi(t)=2 k_{0} L h_{+} \frac{\sin (\Omega T)}{\Omega T} e^{-i \Omega(t-T)},
$$

where following the convention adopted for Eq. (4), $\Delta \phi(t)$ is given by the real part of Eq. (10). For a more detailed discussion of this derivation, see [8].

\section{THE FIELD EQUATIONS IN THE PRESENCE OF THE GW}

We will now show that the same result can be obtained by considering the direct interaction of the gravitational wave with the light propagating in the interferometer arms. In this section we find the equations for the electromagnetic field in the presence of the gw, and in the next section we solve the equations for light propagating in the $x$ and/or $y$ arms. We find the presence of (frequency modulation) sidebands on the carrier displaced by the gw frequency $\Omega$. This is equivalent to the phase shift found in Eq. (10).

In flat space Maxwell's inhomogeneous equations can be written in the manifest covariant form as

$$
\partial_{\mu} F^{\mu \nu}=j^{\nu}
$$

where the field strength tensor is defined as

$$
F_{\mu \nu}=\partial_{\mu} A_{\nu}-\partial_{\nu} A_{\mu}
$$

In a curved space and in the absence of sources, Eq. (11) is replaced by [9]

$$
\partial_{\mu}\left(\sqrt{-g} g^{\mu \lambda} g^{\nu \rho} F_{\lambda \rho}\right)=0
$$


where $g=\operatorname{det}\left(g_{\mu \nu}\right)$ with the field strength tensor still defined by Eq. (12). The dynamical equation (13) can be derived as the Euler-Lagrange equation from the action described by the Lagrangian density

$$
\begin{aligned}
\mathcal{L} & =-\frac{1}{4} \sqrt{-g} g^{\mu \lambda} g^{\nu \rho} F_{\mu \nu} F_{\lambda \rho} \\
& =-\frac{1}{4} \sqrt{-g} g^{\mu \lambda} g^{\nu \rho}\left(\partial_{\mu} A_{\nu}-\partial_{\nu} A_{\mu}\right)\left(\partial_{\lambda} A_{\rho}-\partial_{\rho} A_{\lambda}\right)
\end{aligned}
$$

We will again work in the TT gauge and take the gw to be the same as before. In the TT gauge, in the weak-field approximation $\sqrt{-g}=1$, and keeping only terms linear in $h_{\mu \nu}$, Eq. (13) reads

$$
\partial_{\mu} F^{\mu \nu}-\partial_{\mu}\left(h^{\mu \lambda} F_{\dot{\lambda}}{ }^{\nu}\right)-\partial_{\mu}\left(h^{\nu \rho} F_{. \rho}^{\mu}\right)=0 .
$$

The first term is the same as Eq. (11) with $j^{\nu}=0$, and describes the propagation of the free electromagnetic field. The next two terms act as sources that give rise to new electromagnetic fields generated by the interaction of the gw with the field $F^{\mu \nu}$ of the light circulating in the arms. For the choice of gw (normal incidence toward the negative Z-axis), expansion of Eq. (14) in $\mathbf{E}$ and $\mathbf{B}$ field components yields the four equations

$$
\begin{aligned}
& \nu=0: \quad \nabla \cdot \mathbf{E}-h_{+} e^{-i\left(\Omega t+k_{\Omega} z\right)}\left(\partial_{x} E_{x}-\partial_{y} E_{y}\right) \\
& -h_{\times} e^{-i\left(\Omega t+k_{\Omega} z\right)}\left(\partial_{x} E_{y}+\partial_{y} E_{x}\right)=0, \\
& \nu=1: \quad \partial_{c t} E_{x}-(\nabla \times \mathbf{B})_{x}+h_{+} e^{-i\left(\Omega t+k_{\Omega} z\right)} \\
& \times\left(i \Omega E_{x}+i k_{\Omega} B_{y}-\partial_{c t} E_{x}-\partial_{z} B_{y}\right) \\
& +h_{\times} e^{-i\left(\Omega t+k_{\Omega} z\right)}\left(i \Omega E_{y}+i k_{\Omega} B_{x}-\partial_{c t} E_{y}+\partial_{z} B_{x}\right) \\
& =0 \text {, } \\
& \nu=2: \quad \partial_{c t} E_{y}-(\nabla \times \mathbf{B})_{y}-h_{+} e^{-i\left(\Omega t+k_{\Omega} z\right)} \\
& \times\left(i \Omega E_{y}+i k_{\Omega} B_{x}+\partial_{z} B_{x}-\partial_{c t} E_{y}\right) \\
& -h_{\times} e^{-i\left(\Omega t+k_{\Omega} z\right)}\left(i \Omega E_{x}+i k_{\Omega} B_{y}-\partial_{c t} E_{x}-\partial_{z} B_{y}\right) \\
& =0 \text {, } \\
& \nu=3: \quad \partial_{c t} E_{z}-(\nabla \times \mathbf{B})_{z}+h_{+} e^{-i\left(\Omega t+k_{\Omega} z\right)}\left(\partial_{x} B_{y}+\partial_{y} B_{x}\right) \\
& +h_{\times} e^{-i\left(\Omega t+k_{\Omega} z\right)}\left(\partial_{x} B_{x}-\partial_{y} B_{y}\right)=0 .
\end{aligned}
$$

Since $\Omega \ll \omega_{0}$ we can drop the terms in $\Omega$ and $k_{\Omega}$. We also make use of the condition $z=0$ and choose $h_{+} \neq 0, h_{\times}=0$. This leads to the simplified equations

$$
\begin{array}{ll}
\nu=0: & \boldsymbol{\nabla} \cdot \mathbf{E}=h_{+} e^{-i \Omega t}\left(\partial_{x} E_{x}-\partial_{y} E_{y}\right), \\
\nu=1: & \partial_{c t} E_{x}-(\boldsymbol{\nabla} \times \mathbf{B})_{x}=h_{+} e^{-i \Omega t}\left(\partial_{c t} E_{x}+\partial_{z} B_{y}\right), \\
\nu=2: & \partial_{c t} E_{y}-(\nabla \times \mathbf{B})_{y}=h_{+} e^{-i \Omega t}\left(\partial_{z} B_{x}-\partial_{c t} E_{y}\right), \\
\nu=3: & \partial_{c t} E_{z}-(\nabla \times \mathbf{B})_{z}=-h_{+} e^{-i \Omega t}\left(\partial_{x} B_{y}+\partial_{y} B_{x}\right) .
\end{array}
$$

In the next section we will show how to solve these equations when a plane (light) wave of angular frequency $\omega_{0}$ propagates along the $x$ and/or $y$ axis.

\section{SOLUTION OF THE EQUATIONS OF MOTION}

The electromagnetic (em) fields $\mathbf{E}, \mathbf{B}$ in the arms consist of the carrier field $\mathbf{E}_{0}, \mathbf{B}_{0}$ due to the external source (the laser beam), and the additional field $\mathbf{E}_{+}, \mathbf{B}_{+}$, generated by the source terms in Eq. (17),

$$
\mathbf{E}=\mathbf{E}_{0}+\mathbf{E}_{+}, \quad \mathbf{B}=\mathbf{B}_{0}+\mathbf{B}_{+} .
$$

We describe the carrier as a plane wave propagating along the $x$-axis and polarized along the $z$-axis, and of constant amplitude $A_{0}$

$$
\begin{aligned}
& \mathbf{E}_{0}=A_{0} e^{-i\left(\omega_{0} t-k_{0} x\right)} \mathbf{u}_{z} \\
& \mathbf{B}_{0}=-A_{0} e^{-i\left(\omega_{0} t-k_{0} x\right)} \mathbf{u}_{y}
\end{aligned}
$$

To the sideband fields $\mathbf{E}_{+}, \mathbf{B}_{+}$, we assign slowly timedependent amplitudes $E_{+}(t), B_{+}(t)$ and a phase factor $\left(\omega_{+} t-k_{0} x\right)$ where $\omega_{+}=\omega_{0}+\Omega$, namely,

$$
\begin{aligned}
& \mathbf{E}_{+}=E_{+}(t) e^{-i\left(\omega_{+} t-k_{0} x\right)} \mathbf{u}_{z} \\
& \mathbf{B}_{+}=B_{+}(t) e^{-i\left(\omega_{+} t-k_{0} x\right)} \mathbf{u}_{y} .
\end{aligned}
$$

We will solve the equations in (17) perturbatively noting that $E_{+}, B_{+}$are of order $h$ with respect to $E_{0}, B_{0}$. Thus on the left side of Eq. (17) we must use the full fields $E, B$ but on the right side it suffices to retain only $E_{0}, B_{0}$. With the choice of Eq. (19) for the carrier field only the $\nu=3$ equation is nontrivial; the first three equations are satisfied automatically with the choices made in Eqs. $(19,20)$. Writing out the part of order $h$, for the $\nu=3$ equation in (17), gives

$$
\begin{aligned}
-\frac{\partial E_{+z}}{c \partial t}+\frac{\partial B_{+y}}{\partial x} & =h_{+} e^{-i \Omega t} \frac{\partial B_{0 y}}{\partial x} \\
& =-i h_{+} k_{0} A_{0} e^{-i \omega_{+} t} e^{i k_{0} x}
\end{aligned}
$$

The remaining terms of the equation

$$
-\frac{\partial E_{0 z}}{c \partial t}+\frac{\partial B_{0 y}}{\partial x}=0
$$

describe the propagation of the free carrier field. The fields, both carrier and sideband satisfy Maxwell's dual equation, namely $\partial_{\mu} \tilde{F}^{\mu \nu}=0$. For our choice of direction of propagation and polarization, this condition reduces to

$$
-\frac{\partial E_{+z}}{\partial x}+\frac{\partial B_{+y}}{c \partial t}=0
$$

Combining Eqs. (21) and (23) leads to wave equations for $E_{+z}, B_{+y}$ 


$$
\begin{gathered}
\frac{\partial^{2} E_{+z}}{\partial x^{2}}-\frac{\partial^{2} E_{+z}}{c^{2} \partial t^{2}}=-h_{+} k_{+} k_{0} A_{0} e^{-i\left(\omega_{+} t-k_{0} x\right)} \\
\frac{\partial^{2} B_{+y}}{\partial x^{2}}-\frac{\partial^{2} B_{+y}}{c^{2} \partial t^{2}}=+h_{+} k_{0}^{2} A_{0} e^{-i\left(\omega_{+} t-k_{0} x\right)}
\end{gathered}
$$

where we have introduced $k_{+}=\omega_{+} / c$.

We now use Eqs. (20) and keep in Eqs. (24) only the first time derivatives $d E_{+}(t) / d t, d B_{+}(t) / d t$ of the amplitudes, since $E_{+}(t), B_{+}(t)$ vary slowly. We can also approximate $\omega_{+}^{2} / c^{2}-k_{0}^{2} \simeq 2 \Omega \omega_{0} / c^{2}$ and find the following two eqs for the slowly varying amplitudes

$$
\begin{gathered}
\frac{d E_{+}}{d t}-i \frac{\omega_{0}}{\omega_{+}} \Omega E_{+}=i \frac{h_{+} \omega_{0} A_{0}}{2} \\
\frac{d B_{+}}{d t}-i \frac{\omega_{0}}{\omega_{+}} \Omega B_{+}=-i \frac{h_{+} \omega_{0}^{2} A_{0}}{2 \omega_{+}}
\end{gathered}
$$

with solutions

$$
\begin{array}{r}
E_{+}(t)=\frac{\omega_{+}}{\omega_{0}} \frac{h_{+} \omega_{0} A_{0}}{2 \Omega}\left(e^{i \frac{\omega_{0}}{\omega_{+}} \Omega t}-1\right), \\
B_{+}(t)=-\frac{h_{+} \omega_{0} A_{0}}{2 \Omega}\left(e^{i \frac{\omega_{0}}{\omega_{+}} \Omega t}-1\right) .
\end{array}
$$

where we assumed the initial conditions $E_{+}(t=0)=$ $B_{+}(t=0)=0$. Therefore, to the approximation $\Omega \ll \omega_{0}$ that we are using, the sideband fields are

$$
\begin{gathered}
\mathbf{E}_{+}=\frac{i \omega_{0}}{\Omega} h_{+} A_{0} e^{\frac{i \Omega t}{2}} \sin \frac{\Omega t}{2} e^{-i\left(\omega_{+} t-k_{0} x\right)} \mathbf{u}_{z} \\
\mathbf{B}_{+}=-\frac{i \omega_{0}}{\Omega} h_{+} A_{0} e^{\frac{i \Omega t}{2}} \sin \frac{\Omega t}{2} e^{-i\left(\omega_{+} t-k_{0} x\right)} \mathbf{u}_{y}
\end{gathered}
$$

The amplitudes $E_{+}(t), B_{+}(t)$ of the sideband fields are zero at $t=0$ and grow in time as a parametric amplification process. We will designate them by $A_{+}(t)$. The maximum value of $A_{+}(t)$ is determined by the losses in the arm cavities, however the carrier amplitude, $A_{0}$, remains constant. For one round trip $t=2 L / c=2 T$ and the sideband amplitude has the value

$$
A_{+}(2 T)=i h_{+} L k_{0} A_{0} e^{i \Omega T} \frac{\sin \Omega T}{\Omega T} .
$$

We see that after one round trip the amplitude $A_{0}$ of the carrier acquires a small complex part

$$
A_{+}(2 T)=i A_{0} \phi_{A+}
$$

where

$$
\phi_{A+}=\phi_{A} e^{i \Omega T}=h_{+} k_{0} L \frac{\sin \Omega T}{\Omega T} e^{i \Omega T} \ll 1 .
$$

Thus the amplitude of the propagating light can be written as

$$
A=A_{0}+i A_{0} \phi_{A+} \simeq A_{0} e^{i \phi_{A+}},
$$

which shows that the phase of the carrier is shifted by exactly the same amount as was calculated in section 2 . When the carrier propagates in the $y$-arm the sign of $h$ and thus also of $A_{+}(2 T)$ is reversed; after subtracting the fields returning from the two arms, we find for their relative phase difference $2 \phi_{A+}$, as we had obtained in Eq. (10).

Consider now a gw that depends sinusoidally on time, which we express at $z=0$ by

$$
h_{+}(t)=h_{+} \cos \Omega t=h_{+}\left(\frac{e^{i \Omega t}+e^{-i \Omega t}}{2}\right) .
$$

It is clear that in this case both an upper and lower sideband will be present. The fields are

$E_{+}=\frac{i E_{0} \phi_{A+}}{2} e^{-i\left(\omega_{+} t-k_{0} x\right)}, \quad E_{-}=\frac{i E_{0} \phi_{A-}}{2} e^{-i\left(\omega_{-} t-k_{0} x\right)}$,

where $\phi_{A-}=\phi_{A+}^{*}=\phi_{A} e^{-i \Omega T}$; to see this change $\Omega \rightarrow-\Omega$ in Eq.(30). At $t=0, x=0$ the two sideband fields have equal imaginary parts and opposite real parts. This is the condition that corresponds to phase (or frequency) modulation when the sidebands are combined with the carrier

$$
\begin{aligned}
E & =E_{0} e^{-i\left(\omega_{0} t-k_{0} x\right)}\left(1+\frac{i \phi_{A+}}{2} e^{-i \Omega t}+\frac{i \phi_{A-}}{2} e^{i \Omega t}\right) \\
& =E_{0} e^{-i\left(\omega_{0} t-k_{0} x\right)}\left[1+i \frac{\phi_{A}}{2}\left(e^{-i \Omega(t-T)}+e^{i \Omega(t-T)}\right)\right] \\
& \simeq E_{0} e^{-i\left[\omega_{0} t-\phi_{A} \cos \Omega(t-T)-k_{0} x\right]} .
\end{aligned}
$$

In conclusion a sinusoidal gw interacting with a plane wave carrier (under the appropriate geometry) imposes upper and lower sidebands, or equivalently contributes a time-dependent phase shift. We obtained this result by considering the propagation of the carrier in the spacetime of the gw, without referring to the change in the round-trip time of travel to and from the end mirror.

\section{DISCUSSION}

Our derivation is based on a subtle point, the use of the TT gauge. We can use the TT gauge only because the end points of the light travel (the mirrors) are free. If the mirrors were fixed we would have to do the calculation in the LL gauge, i.e. in the laboratory frame of reference. In the LL gauge [10 13] the amplitude of the gw is modified from the form given by Eq. (4), the relevant part of the metric being 


$$
h_{00}=\frac{1}{2 c^{2}} \ddot{h}(t)\left(x^{2}-y^{2}\right)
$$

For low frequency gw's the resulting phase shift when the mirrors are fixed is much smaller than for free mirrors, and this is why interferometric gw detectors are constructed with suspended mirrors. For the details of the calculation in the LL gauge see ref. [1].

The presence of sidebands has a direct physical interpretation in terms of absorption and stimulated emission of gravitons from/to the gw. Since the gw field is highly classical, [the occupation number for $h \sim 10^{-23}$ and $f \sim 100 \mathrm{~Hz}$, is $\left.n=N_{g} /(\lambda / 2 \pi)^{3} \sim 10^{33}\right]$, both processes have the same probability. Absorption leads to the upper sideband, while emission to the lower one. There is no energy exchange between the optical and gravitational fields, but only a phase shift.

In our analysis we have assumed that the carrier is a plane wave propagating toward the positive $\mathrm{x}$-direction, see Eqs. (19). In practice the carrier is reflected by a mirror at $x=a$, thus the Electric field should vanish at $x=a$. This condition is satisfied by writing the fields in the form

$$
\begin{aligned}
& \mathbf{E}_{0}=A_{0}\left[e^{-i\left(\omega_{0} t-k_{0} x\right)}-e^{-i\left(\omega_{0} t+k_{0} x-2 k_{0} a\right)}\right] \mathbf{u}_{z}, \\
& \mathbf{B}_{0}=-A_{0}\left[e^{-i\left(\omega_{0} t-k_{0} x\right)}+e^{-i\left(\omega_{0} t+k_{0} x\right)-2 k_{0} a}\right] \mathbf{u}_{y} .
\end{aligned}
$$

If a mirror is also placed at $x=0$ to reflect the carrier and $k_{0} a=n \pi$, a standing wave is established in the region $0 \leqq x \leqq a$. This does not modify the conclusions that we have reached. The case when the carrier is a standing wave is treated by Cooperstock and Faraoni 14] and leads to the same results as in Eq. (34). The interaction of an electromagnetic and gravitational field was first discussed by Gertsenshtein and Pustovoit 15] in 1963, and subsequently by others [12, 16 18].

We have also shown that a gw will couple to the carrier in a single arm. The interferometer configuration has been chosen because it is technically advantageous. The light returning from the two arms is adjusted to interfere destructively at the detection point in the absence of a gw. Thus when the gw induces a phase shift, a signal appears over a null background (excluding noise). By using multiple traversals in the arms one can increase the effective length, and thus the phase shift, significantly. The main limitation in effective arm length (apart from losses in the optics) is related to the frequency of the gw. We have seen in Eq. (10) that the phase shift is modulated by the "form factor" $\sin (\Omega T) / \Omega T$, and therefore $\Omega L / c$ must be kept small.

We thank Dr. R. Weiss for bringing to our attention ref. [14]. This work was supported in part by DOE Grant DE-FG02-91ER40685 and NSF Grant PHY-0456239.
[1] R. Weiss, Quarterly Progress Report no 105, RLE-MIT (1972); A. Abramovici et al., Science 256, 325 (1992).

[2] R. L. Forward, Phys. Rev. D17, 379, (1978).

[3] P. Linsay, P. Saulson, R. Weiss and S. Whitcomb, "A Study of a Long Baseline Gravitational Wave Antenna System", October 1983, Prepared for the National Science Foundation.

[4] P. Saulson, Fundamentals of Interferometric Gravitational Wave Detectors, World Scientific, Singapore (1994).

[5] E. D. Black and R. N. Gutenkust, Am. J. Phys. 71, 365 (2003).

[6] P. Saulson, Am. J. Phys. 65, 501 (1997).

[7] C. W. Misner, K. S. Thorne and J. A. Wheeler, Gravitation W.H.Freeman, NY (1973).

[8] M. Rakhmanov, Class. Quantum Grav. 26, 155010 (2009).
[9] L. D. Landau and E. M. Lifshitz, The Classical Theory of Fields, 2nd edition Adison-Wesley, Boston (1962).

[10] R. Blanford and K. S. Thorne, Ph 136: Applications of Classical Physics, Chapter 26. California Institute of Technology, (2003).

[11] M. Rakhmanov, Phys. Rev. D71, 084003 (2005).

[12] F. Pegoraro, E. Picasso and L. A. Radicati, J.Phys. A11, 1949 (1978).

[13] S. B. Tarabrin, Phys. Rev. D75, 102002 (2007).

[14] F. I. Cooperstock and V. Faraoni, Class. Quantum Gravity 10, 1189 (1993).

[15] M. E. Gerstenshtein and V. I. Pustovoit, Soviet Physics, JETP 16, 433 (1963).

[16] C. M. Caves, Phys. Lett. 90B, 323 (1979).

[17] F. I. Cooperstock, Foundations of Physics 22, 1011 (1992).

[18] J. A. Lobo, Class. Quantum Grav. 9, 1385 (1992). 